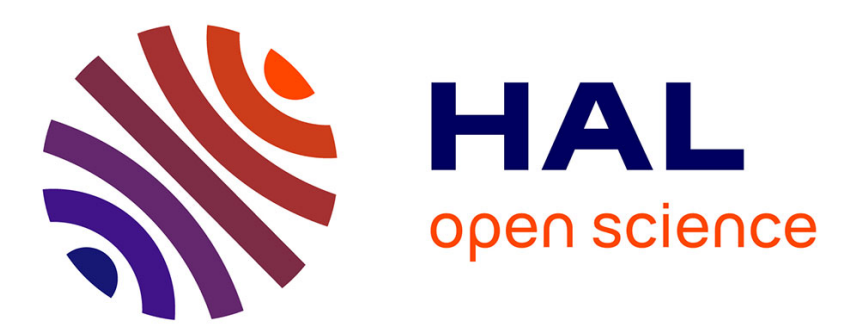

\title{
A biophysical approach to Phospholipase A activity and inhibition by anti-inflammatory drugs
}

Diana Gaspar, Marlene Lúcio, Kerstin Wagner, Gerald Brezesinski, Sandra Rocha, J.L.F. Costa Lima, Salette Reis

\section{- To cite this version:}

Diana Gaspar, Marlene Lúcio, Kerstin Wagner, Gerald Brezesinski, Sandra Rocha, et al.. A biophysical approach to Phospholipase A activity and inhibition by anti-inflammatory drugs. Biophysical Chemistry, 2010, 152 (1-3), pp.109. 10.1016/j.bpc.2010.08.006 . hal-00694282

\section{HAL Id: hal-00694282 \\ https://hal.science/hal-00694282}

Submitted on 4 May 2012

HAL is a multi-disciplinary open access archive for the deposit and dissemination of scientific research documents, whether they are published or not. The documents may come from teaching and research institutions in France or abroad, or from public or private research centers.
L'archive ouverte pluridisciplinaire HAL, est destinée au dépôt et à la diffusion de documents scientifiques de niveau recherche, publiés ou non, émanant des établissements d'enseignement et de recherche français ou étrangers, des laboratoires publics ou privés. 


\section{Accepted Manuscript}

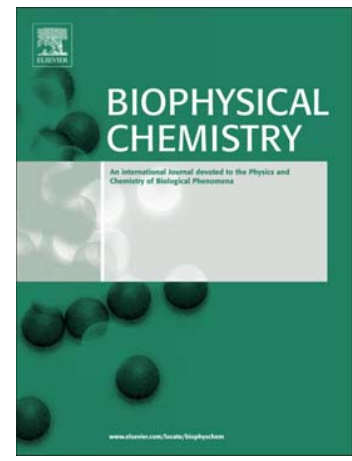

A biophysical approach to Phospholipase $\mathrm{A}_{2}$ activity and inhibition by anti-inflammatory drugs

Diana Gaspar, Marlene Lúcio, Kerstin Wagner, Gerald Brezesinski, Sandra Rocha, J.L.F. Costa Lima, Salette Reis

PII: $\quad$ S0301-4622(10)00220-6

DOI: $\quad$ doi: $10.1016 /$ j.bpc.2010.08.006

Reference: $\quad$ BIOCHE 5422

To appear in: Biophysical Chemistry

Received date: 16 May 2010

Revised date: $\quad 14$ August 2010

Accepted date: 17 August 2010

Please cite this article as: Diana Gaspar, Marlene Lúcio, Kerstin Wagner, Gerald Brezesinski, Sandra Rocha, J.L.F. Costa Lima, Salette Reis, A biophysical approach to Phospholipase $\mathrm{A}_{2}$ activity and inhibition by anti-inflammatory drugs, Biophysical Chemistry (2010), doi: 10.1016/j.bpc.2010.08.006

This is a PDF file of an unedited manuscript that has been accepted for publication. As a service to our customers we are providing this early version of the manuscript. The manuscript will undergo copyediting, typesetting, and review of the resulting proof before it is published in its final form. Please note that during the production process errors may be discovered which could affect the content, and all legal disclaimers that apply to the journal pertain. 


\section{A biophysical approach to Phospholipase $A_{2}$ activity and inhibition by anti- inflammatory drugs}

Diana Gaspar ${ }^{\mathrm{a}}$, Marlene Lúcio ${ }^{\mathrm{a}}$, Kerstin Wagner ${ }^{\mathrm{b}, \mathrm{c}}$, Gerald Brezesinski ${ }^{\mathrm{c}}$, Sandra Rocha ${ }^{\mathrm{d}}$, J.L.F. Costa Lima ${ }^{\mathrm{a}}$ and Salette Reis ${ }^{\mathrm{a}^{*}}$

a REQUIMTE, Departamento de Química, Faculdade de Farmácia, Universidade do Porto, Rua Aníbal Cunha 164, 4090-030 Porto, Portugal bresent address: MEMPHYS - Center for Biomembrane Physics, University of Southern Denmark, 5230 Odense M, Denmark

${ }^{\mathrm{c}}$ Max - Planck Institute of Colloids and Interfaces, Science Park Golm, Am Mühlenberg 1, D14476 Potsdam, Germany

${ }^{\mathrm{d}}$ LEPAE, Chemical Engineering Department, Faculty of Engineering, University of Porto, R. Roberto Frias 4200-465 Porto, Portugal

*Corresponding author: Salette Reis

Departamento de Química, Faculdade de Farmácia, Universidade do Porto Rua Aníbal Cunha, 164

4099-030 Porto

Portugal

Tel: $+351-222078966$

Fax: +351-222078961

Email: shreis@ff.up.pt 


\section{ABSTRACT}

The present study describes the interaction of two nonsteroidal anti-inflammatory drugs (ibuprofen and piroxicam) with $\mathrm{PLA}_{2}$ from Naja mossambica mossambica and seeks to deepen the knowledge about the influence of the biophysical properties of biomembranes, and the inhibitory effect of the drugs on the enzymatic activity. Fluorescent techniques with and without the use of probes, surface pressure/molecular area isotherms, surface pressure/time and molecular area/time measurements combined with circular dichroism spectroscopy and direct techniques of visualization of lipid membranes (Brewster angle microscopy), revealed that both drugs inhibit $\mathrm{PLA}_{2}$. Additionally, the structure and characteristics of the lipid bilayer, as well as, the direct interaction of drugs with the enzyme seem to play an important role on the hydrolytic activity of PLA $_{2}$ towards membrane model systems. These results open a way of finding new and better strategies that can contribute to the development of suitable agents for relieving inflammatory conditions. 


\section{Graphical abstract}

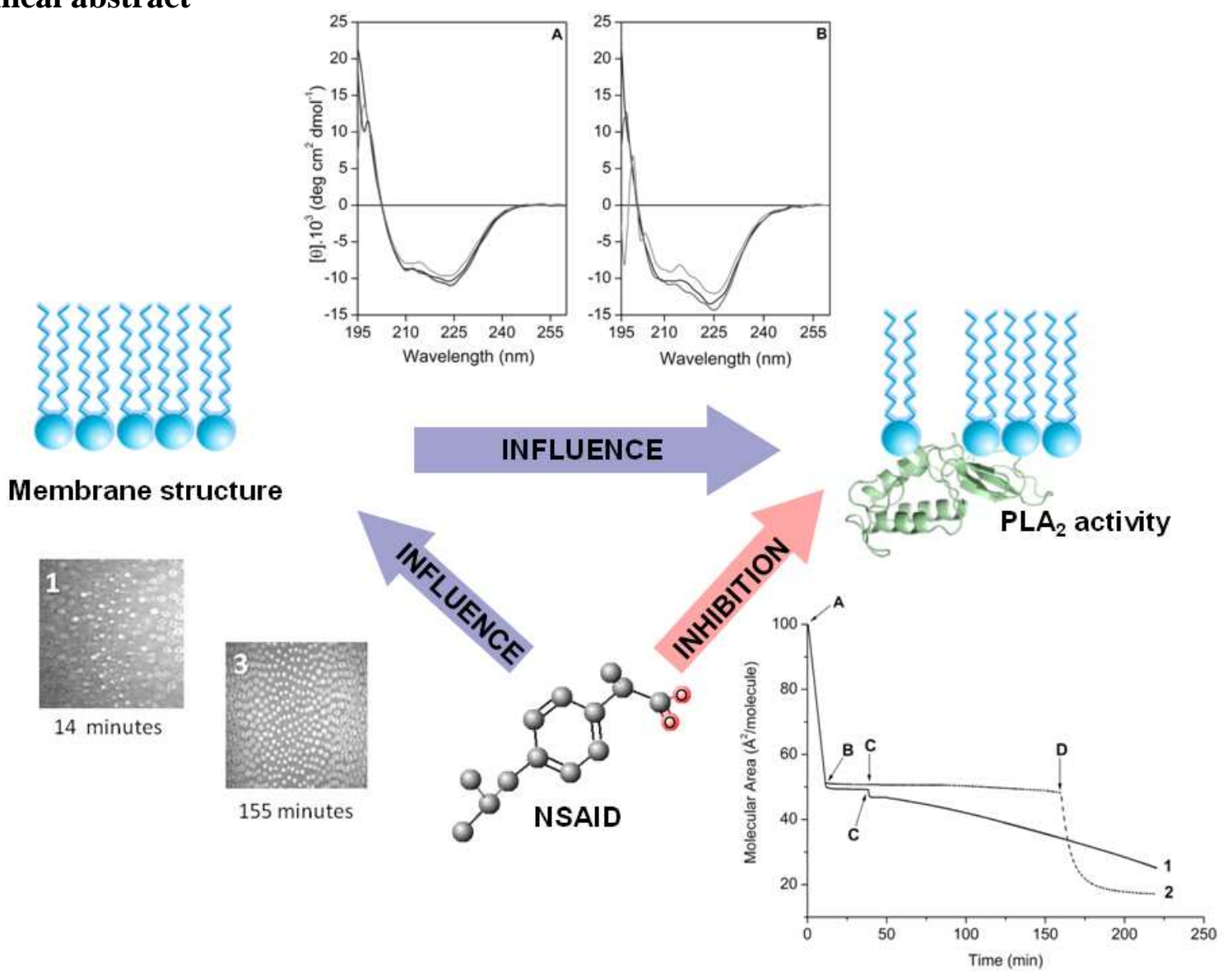

Keywords: PPLA $_{2}$; NSAIDs; enzymatic inhibition; membrane model systems; membrane biophysical properties 


\section{Introduction}

Phospholipase $\mathrm{A}_{2}\left(\mathrm{PLA}_{2}\right)$ is a water-soluble enzyme responsible for the hydrolysis of phospholipids on the sn-2 acyl bond with the consequent release of free fatty acids and lysophospholipids [1]. Arachidonic acid (AA) is among the free fatty acids released representing a possible store of energy [2] and being involved in the biosynthesis of prostaglandins, prostacyclins, thromboxanes and interleukins - eicosanoids, known as mediators of inflammation and signal transduction [2-4]. Plants, mammals, snakes and bee venoms can be the source of $\mathrm{PLA}_{2}$ and according to their cellular location, these enzymes can be classified as cytosolic $\left(\mathrm{cPLA}_{2}\right)$, calcium independent $\left(\mathrm{iPLA}_{2}\right)$ and secretory $\left(\mathrm{sPLA}_{2}\right)[5]$. Considering the three types of $\mathrm{PLA}_{2}$ mentioned, studies revealed that $\mathrm{SPLA}_{2}$ is deeply involved in the inflammatory process of several conditions $[3,6]$ such as rheumatoid arthritis, atherosclerosis, respiratory distress syndrome and septic shock [4, 5, 7]. Most precisely, sPLA $_{2}$ leads to the formation of lipid inflammatory mediators through its lipolytic activity and also by activation of $\mathrm{PLA}_{2}$ [7].These findings imply that regulation of $\mathrm{PLA}_{2}$ activity, and in particular sPLA 2 activity, is a potential key for controlling inflammatory diseases.

In the presented work, the interaction between two non-steroidal anti-inflammatory drugs (NSAIDs) with SPLA $_{2}$ from Naja mossambica mossambica venom and model membranes was investigated. The choice of this enzyme was based on the large similarity in structure and catalytic functions with mammalian enzymes [8]. Since lipids are the major components of biological membranes and are essential in the regulation of enzymes, lipid model systems were used herein to mimic membranes and to study PLA 2 activity. Indeed, PLA 2 activity is conditioned by the membrane structure [9] and enhanced in presence of phospholipid substrates in the aggregated form [10] such as liposomes and monolayers. In this regard, monolayers used as the half of the membrane bilayers leaflet, provide an adequate system for the investigation of the effects exerted on $\mathrm{PLA}_{2}$ activity as a consequence of the membrane structure. These model systems also allow controlling the experiments conditions such as the 
lipid and subphase composition [11]. Besides monolayers, liposomes were also used as representatives of the membrane lipid bilayers. Furthermore, the lipid composition of the model membranes was chosen to promote interfacial activation of $\mathrm{PLA}_{2}$ activity. In fact it has been observed a substantial increase in the enzymatic activity upon the control of some key properties of the lipid substrate, which include curvature, structural defects, phase separation, surface charge and others $[9,12]$,. In this context a lipid mixture (7:3) of 1, 2-dimyristoyl-snglycero-3-phosphocholine (L-DMPC) and 1, 2-dipalmitoyl-sn-glycero-3phosphoethanolamine (L-DPPE) was used. This lipid mixture was chosen based on the fact that snake venom $\mathrm{PLA}_{2}$ activity can be enhanced by zwitterionic phospholipids [13], and that the presence of lipid domains (de-mixing) results in the adequate environment for PLA 2 hydrolysis [9] [14]. Accordingly, the phospholipids DMPC and DPPE are zwitterionic at the $\mathrm{pH}$ of the studies (physiological $\mathrm{pH} 7.4$ ). Furthermore, at the temperature of studies $\left(37^{\circ} \mathrm{C}\right)$, the phase diagram of the aqueous dispersions of the chosen lipid binary system assures the existence of gel-fluid heterogeneity important to activate PLA ${ }_{2}[15]$ The NSAIDs studied, piroxicam and ibuprofen, act as anti-inflammatory drugs by reducing the formation of inflammatory chemical mediators such as prostaglandins and leukotrienes [16]. Another possible target for the anti-inflammatory action is $\mathrm{PLA}_{2}$, which makes the NSAIDs studied potential inhibitors of this enzyme. In fact, several studies report the inhibition of $\mathrm{PLA}_{2}$ by NSAIDs. For instance, it has been shown that indomethacin and alminoprofen inhibit rat peritoneal and snake venom $\mathrm{PLA}_{2}$, respectively [17]. Other molecules, like $\alpha$-lipoic acid (ALA), inhibit several sPLA 2 from human serum and also from snake venoms [3]. Although the mechanism for this inhibition is still unclear, it seems that while some molecules change the membrane biophysical properties, blocking the passage and the contact of PLA 2 with the membrane [3], others have a direct contact with the enzyme and can also form complexes with it, as demonstrated in studies involving ALA [3] and oxyphenbutazone [1]. In view to 
this, the experiments presented in this work have first the purpose to study the PLA 2 activity and inhibition by the NSAIDs tested, and second, to determine the factor(s) that contribute to that inhibition which might include: (i) the drug induced biophysical changes occurring at the interface where PLA 2 activity takes place; (ii) the direct interaction between the drug and enzyme and/or the drug induced disturbance in the binding of $\mathrm{PLA}_{2}$ to the substrate. To achieve these purposes, spectrofluorimetric techniques regarding enzymatic activity/inhibition and binding studies were combined with biophysical studies, circular dichroism spectroscopy and Brewster angle microscopy (BAM) as a direct technique of visualization of lipid membranes. Therefore, this work represents a systematic study of evaluation of PLA 2 inhibitors, covering not only the direct inhibitory assays and the induced changes in enzyme's conformation, but also assays that evaluate the role of the membrane biophysical changes induced by drugs on the enzymatic activity. In this context, this study, contributing to identify drugs-induced alterations on membrane lipid physical properties and enzymatic conformation putatively correlated with $\mathrm{PLA}_{2}$ inhibitory activity, may provide significant insights for predicting or modulating the impact of other related compounds, potentially used for pharmacological purposes, since they may be significant to the development of novel inhibitors with improved anti-inflammatory properties.

\section{Material and Methods}

\subsection{Materials}

Non steroidal anti-inflammatory drugs, ibuprofen and piroxicam, 1, 2-dimyristoyl-sn-glycero3-phosphocholine (L-DMPC), 1, 2-dipalmitoyl-sn-glycero-3-phosphoethanolamine (L-DPPE) and lyophilized powder of Naja mossambica mossambica phospholipase $\mathrm{A}_{2}$ were purchased from Sigma-Aldrich. AcryloDated Intestinal Fatty Acid Binding protein (ADIFAB) was from Molecular Probes. All reagents were used without further purification. 
Solutions of ADIFAB and anti-inflammatory drugs were prepared in Tris buffer (Tris $10 \mathrm{mM}$; $\mathrm{NaCl} 150 \mathrm{mM} ; \mathrm{CaCl}_{2} 1 \mathrm{mM}$; pH 7.4). Solutions were prepared with double-deionised water (conductivity less than $0.1 \mu \mathrm{S} \mathrm{cm}^{-1}$ ).

\subsection{ADIFAB based fluorescence assay}

To monitor the $\mathrm{PLA}_{2}$ activity, a fluorescence methodology was applied using ADIFAB as a fluorescence probe [18]. ADIFAB is a conjugate of the polarity-sensitive fluorescent probe acrylodan and intestinal fatty acid binding protein (I-FABP), which has a high binding affinity for free fatty acids (FFA) resultant from the lipid hydrolysis catalyzed by PLA2. Detection of FFA by ADIFAB is based on a change in the position of the acrylodan fluorophore relatively to the nonpolar binding pocket of the protein when it becomes occupied by a fatty acid (FA). The fluorescence intensities at $505 \mathrm{~nm}$ and $432 \mathrm{~nm}$ corresponding to the bound and unbound probe, as well as single wavelength measurements at $432 \mathrm{~nm}$, allow the determination of FFA released from the liposomes and of the total fatty acid concentration [19].

The experiments were carried out using DMPC+DPPE (7:3) LUVs as PLA 2 substrate. The chosen phospholipids were co-dissolved in chloroform, mixed and dried under nitrogen stream. The resultant lipid film was left under vacuum for complete solvent removal and then dispersed in Tris buffer (Tris $10 \mathrm{mM}, \mathrm{NaCl} 150 \mathrm{mM}, \mathrm{CaCl}_{2} 1 \mathrm{mM}, \mathrm{pH} 7.4$ ) and vortexed to obtain multilamellar vesicles (MLVs). The MLVs suspension was extruded 10 times in a extruder device (LIPEX ${ }^{\mathrm{TM}}$ extruder from Northern Lipids Inc) through polycarbonate filters with a pore diameter of $100 \mathrm{~nm}$ to form unilamellar vesicles (LUVs). Both dispersion in Tris buffer and extrusion were performed above the phase transition temperature of the lipid mixture $\left(55^{\circ} \mathrm{C}\right)$. 
The reaction mixture of a total volume of $1200 \mu \mathrm{L}$ was prepared in $1 \mathrm{~cm}$ path length quartz cuvette containing Tris buffer (Tris $10 \mathrm{mM}, \mathrm{NaCl} 150 \mathrm{mM}, \mathrm{CaCl}_{2} 1 \mathrm{mM}, \mathrm{pH} 7.4$ ), $50 \mu \mathrm{M}$ liposomes of DMPC and DPPE (7:3), ADIFAB $0.108 \mu \mathrm{M}$, Naja mossambica mossambica $\mathrm{PLA}_{2} 7.6 \times 10^{-10} \mathrm{M}$ and NSAID $0-20 \mu \mathrm{M}$ (in the assays attained for studying the PLA 2 enzymatic inhibition by piroxicam and ibuprofen) or buffer (in the control assays). The lipid hydrolysis reaction was monitored in a Perkin-Elmer LS 50B spectrofluorimeter with excitation wavelength set at $386 \mathrm{~nm}$ and emission wavelength at $432 \mathrm{~nm}$. Fluorescence values were corrected for light scattering contributions by subtraction of intensities from unlabeled samples at the same conditions. These contributions were always negligible (less than $0.5 \%$ ). The assays performed for studying the enzymatic inhibition by piroxicam and ibuprofenrequired an incubation period of 30 minutes after which the $\mathrm{PLA}_{2}$ - drug mixture was added to the lipid suspension. The $\mathrm{PLA}_{2}$ activity obtained without the drug in the control assay was considered to be $100 \%$. All the other activities measured with the PLA $_{2}$-drug mixture were compared to the one without drug and considered as relative percentages. The temperature of the measurements was kept constant at $37^{\circ} \mathrm{C}$ by an external bath circulator.

\subsection{Langmuir isotherms}

To study the interaction of the NSAIDs with the lipid monolayers, surface pressure/time $(\pi / t)$ measurements and surface pressure/molecular area $(\pi / \mathrm{A})$ isotherms were performed in a Langmuir trough.

Monolayers of the lipid mixture DMPC/DPPE (7:3) were obtained after spreading phospholipid /chloroform solutions $(1 \mathrm{mM})$ on a Tris buffer subphase (Tris $10 \mathrm{mM}, \mathrm{NaCl} 150$ $\left.\mathrm{mM}, \mathrm{CaCl}_{2} 1 \mathrm{mM}, \mathrm{pH} 7.4\right)$ or on ibuprofen/piroxicam subphases $(20 \mu \mathrm{M})$ prepared in the same buffer. After waiting 10 minutes for evaporation of the solvent, isotherms were recorded on a film balance from $\mathrm{R} \& \mathrm{~K} \mathrm{GmbH}$ (Potsdam, Germany), equipped with a Wilhelmy-type 
pressure-measuring system using a filter paper plate. Initially, $\pi / \mathrm{t}$ dependecies were measured until a stabilized surface pressure was reached, to assure that the adsorption of NSAIDs to the monolayer reached a steady-state. At this point, $\pi / \mathrm{A}$ isotherms were measured by the compression of the monolayers at a rate of $5 \AA^{2} /$ molecule/min.

Langmuir trough was also used to study the lipid hydrolysis efficiency of $\mathrm{PLA}_{2}$ and its inhibition by the NSAIDs studied. In this case, monolayers of DMPC/DPPE (7:3) were obtained in a similar way as described above on a Tris buffer subphase (Tris $10 \mathrm{mM}, \mathrm{NaCl}$ $150 \mathrm{mM}, \mathrm{CaCl}_{2} 1 \mathrm{mM}, \mathrm{pH}$ 7.4). Initially, the monolayer was compressed until a desired surface pressure was reached. At this point, the compression was stopped, and the monolayer was left for stabilization until the molecular area has reached a constant value. After this, A / $\mathrm{t}$ dependencies were plotted using two different types of incubation procedures. In the first procedure, $\mathrm{PLA}_{2}(4 \mu \mathrm{M})$ was injected into the subphase and the variation of the molecular area over time was recorded. The final concentration of $\mathrm{PLA}_{2}$ in the subphase was $0.4 \mathrm{nM}$. In the second procedure, $\mathrm{PLA}_{2}(4 \mu \mathrm{M})$ and drug $(50 \mathrm{nM})$ were incubated for 30 minutes after which the mixture was injected into the subphase and the variation of the molecular area over time was recorded. More than $120 \mathrm{~min}$ after this first injection, $1 \times 10^{-3} \mathrm{M}$ of $\mathrm{PLA}_{2}$ was injected into the subphase, and the variation of the molecular area was once again registered.

\subsection{Brewster Angle Microscopy (BAM)}

Morphological images of the monolayers were obtained with a Brewster angle microscope; model BAM1, NFT - Nanofilm Technologie GmbH, Goettingen, Germany, settled up onto the computer-interfaced Langmuir trough. The microscope was equipped with a He-Ne laser (632.8 nm, $10 \mathrm{~mW})$, a polarizer, an analyzer and a CCD camera. BAM images were recorded on a video tape and then transferred to a personal computer [20] [21]. 


\subsection{Fluorescence binding measurements}

The intrinsic fluorescence intensity of $\operatorname{PLA}_{2}(0.36 \mu \mathrm{M})$ was measured in the presence of several concentrations of piroxicam and ibuprofen $(0-150 \mu \mathrm{M})$. All measurements were carried out in a Perkin-Elmer LS 50B spectrofluorimeter. Excitation and emission wavelength were set at $220 \mathrm{~nm}$ and $345 \mathrm{~nm}$, respectively. Fluorescence emission spectra were obtained between 275 and $410 \mathrm{~nm}$. All fluorescence intensity data were corrected from reabsorption and inner filter effects [22]. The temperature of all measurements was kept constant at $37{ }^{\circ} \mathrm{C}$ with an external bath circulator.

\subsection{Circular dichroism (CD) spectroscopy}

CD spectra were obtained on a JASCO J-715 Spectropolarimeter (Jasco Co., Tokyo, Japan) using a cuvette with $1 \mathrm{~mm}$ path length. Data were collected at $0.2 \mathrm{~nm}$ intervals from 195 to $260 \mathrm{~nm}$ for wavelength scans, with the average of ten scans recorded at a speed of $50 \mathrm{~nm} / \mathrm{min}$. To evaluate the enzyme conformational changes due to membrane binding, CD spectra of $\mathrm{PLA}_{2}\left(8.9 \mu \mathrm{M}\right.$ ) were measured in Tris buffer (Tris $10 \mathrm{mM}, \mathrm{NaCl} 150 \mathrm{mM}, \mathrm{CaCl}_{2} 1 \mathrm{mM}, \mathrm{pH}$ 7.4) and after incubation with LUVs of DMPC+DPPE (7:3) $(430 \mu \mathrm{M})$. Similar measurements were performed for the drugs tested, piroxicam and ibuprofen, in buffer or in lipid media. To study the effect of drugs on $\mathrm{PLA}_{2}$ structure in presence of DMPC+DPPE liposomes, the samples were incubated by two different procedures. In one case, the enzyme was first mixed with piroxicam or ibuprofen $(77.5 \mu \mathrm{M})$ and then added to the liposome suspension. In a different approach, drugs were first incubated with liposomes and the enzyme solution was added afterwards. The protein spectra were corrected by subtracting the appropriate blank runs (buffer, drug and/or liposomes in buffer) and converted to mean residue molar ellipticity, represented by $[\theta]$. Spectra were smoothed using the noise reduction routines provided with the $\mathrm{J}-715$ spectropolarimeter 


\section{Results and Discussion}

Biophysical studies concerning $\mathrm{PLA}_{2}$ activity led to the assumption that the reaction taking place at the interface can be visualized as two distinct steps, interfacial recognition and catalytic cleavage $[10,23]$ and that the enzymatic activity is enhanced in the presence of aggregated phospholipids, e.g., monolayers at the air/water interface [23]. The explanation regarding this behavior is covered by two theories that should be considered simultaneously: the enzyme theory, where $\mathrm{PLA}_{2}$ undergoes a conformational change favorable to the reaction, and the substrate theory, where the characteristics of the substrate (like molecular orientation and fluidity) have an important role in the course of the reaction [10]. To understand the mechanism underlying the inhibition of $\mathrm{SPLA}_{2}$ by piroxicam and ibuprofen, it is necessary to determine whether the drugs are responsible for changes in the lipid structure and lipid packing leading to the inability of $\mathrm{PLA}_{2}$ to access the substrate, or if the drugs show a direct interaction with the enzyme inhibiting its activity. Regarding these aims, different studies were made to understand if the inhibitory effect of the NSAIDs studied is related with their action on the membrane structure and/or their direct binding to $\mathrm{PLA}_{2}$

\subsection{Studies of membrane biophysical changes induced by NSAIDs}

The adsorption behaviour of piroxicam and ibuprofen to a monolayer of DMPC/DPPE $(7: 3)$ was the first step for assessing the effect of the drugs on membrane structure. During the adsorption process of the drugs to the monolayer it was possible to see a time-dependent increase of the pressure $(\pi)$ as a result of the insertion of the drug molecules into the lipid monolayer. The adsorption was complete only after 4-6 hours, which was the time needed to achieve the steady-state pressure. The steady-state pressure reached for the same NSAID concentration was $10 \mathrm{mN} / \mathrm{m}$ and $4.5 \mathrm{mN} / \mathrm{m}$ for piroxicam and ibuprofen, respectively. The 
observed surface pressure increase indicates that both NSAIDs are able to adsorb/penetrate to the monolayer and that this effect is more pronounced in the case of piroxicam. Similar effect of ibuprofen on monolayers of DPPC has been shown recently [24]. Indeed, Jablonowska and coworkers demonstrated that when ibuprofen is present in the subphase the organization of molecules into a liquid monolayer starts immediately at larger areas per molecule corresponding to an earlier onset of the increase of surface pressure. However, in the current study, the NSAIDs' time dependent interaction with lipid component of membranes was also assessed, since this aspect is most times overlooked. Therefore, it was possible to conclude that there is a time-dependent increase of the surface pressure until a steady-state was reached where the drug adsorption/penetration into the monolayers was considered to reach its maximum. It is thus interesting to notice that similar effects obtained by other authors for higher concentrations of ibuprofen [24], where attained here for smaller concentrations of the same drug but with larger times of interaction with the lipid component.The NSAIDs' adsorption process to the lipid monolayers was also followed visually by BAM. This technique takes advantage of the Brewster angle, 53.1 , where no reflection arises from the interface after it has been illuminated with p-polarized light. Consequently, when a monolayer is present at the interface it causes a change in the Brewster angle conditions by changing the refractive index, thus reflecting p-polarized light [23]. This permits the visualization of domains arising during first-order phase transitions between fluid and condensed phases or due to de-mixing into fluid and condensed regions. In the case of the interface studied, BAM images show a darker area representing the fluid phase (DMPC) and bright spots correspondent to condensed domains (phase-separated DPPE). A comparison of BAM images obtained at different points of the adsorption process of the drugs (as can be seen in Figure 1 for piroxicam) shows that the penetration of the drug molecules into the phospholipid layer results in changes in the membrane domains with different contrast effects. At the steady-state 
pressure where the drug shows the highest adsorbtion to the monolayer, there is a well defined contrast between a fluid dark area correspondent to the membrane fluid phase and brighter areas correspondent to spherical condensed domains (Figure 1 - number 3). The size of the condensed phase domains is not changed, but the density is drastically increased.

After the adsorption process has reached the maximum pressure, the monolayer was compressed and $\pi / \mathrm{A}$ isotherms were plotted. Two types of $\pi / \mathrm{A}$ isotherms were measured from the monolayer compression on a buffer subphase with or without the NSAIDs tested. The resultant isotherms are presented in Figure 2.

As a reference, the $\pi$ / $\mathrm{A}$ isotherm of the lipid mixture in the absence of drugs (Figure 2 A-line 1) showed an expanded state, with no evidence of any phase transition. The condensed phase domains are observable already at large molecular areas indicating that a phase-separation into a liquid phase, mainly composed of DMPC, and a condensed phase, mainly composed of DPPE, occurs. From the extrapolation of the steeper portion of the isotherm slope to zero surface pressure, it is possible to obtain the limiting molecular area $\mathrm{A}_{0}\left(\approx 6.5 \mathrm{~nm}^{2} /\right.$ molecule $)$ of the mixture which is in good agreement with values reported for DMPC monolayers [25]. Indeed, it has also been reported that the effective cross-sectional area of DMPC in a mixed monolayer is similar to the area occupied by a DMPC molecule in a tightly packed monolayer [26], and thus the small amount of condensed DPPE does not seem to have great influence on the limiting molecular area of the studied monolayer.

The addition of both NSAIDs to the subphase changes the $\pi / \mathrm{A}$ isotherms revealing the penetration of the drugs which now occupy parts of the surface area of the trough. At large molecular areas of the lipid mixture, the drug penetration leads to the same pressure as observed for the $\pi / \mathrm{t}$ measurements. On compression, the drugs are progressively squeezed out from the lipid monolayer and at higher lateral pressures one observes the same molecular 
area as for the pure lipid monolayer. Although these common effects were found for both NSAIDs, isotherms with different profiles were obtained for each one of these drugs. At larger surface areas, piroxicam revealed higher penetration since the isotherm starting pressure value was greater than the observed for ibuprofen (Figure $2 \mathrm{~A}$ - lines 3 and 2). However, with increasing compression ibuprofen revealed a shift of the isotherm to higher surface pressures indicating that this NSAID is better stabilized in the lipid layer compared to piroxicam. This isotherm shift caused by ibuprofen decreases with the onset of a plateau at 18 $\mathrm{mN} / \mathrm{m}\left(73 \AA^{2} /\right.$ molecule) indicating a partial expulsion of the drug. At the end of this plateau, the isotherms from the lipid mixture on the pure buffer solution and on the buffer containing ibuprofen almost superimpose (Figure $2 \mathrm{~A}$ - line 2).

The described observations support the assumption that piroxicam is more surface active whereas ibuprofen is better stabilized in a lipid monolayer.

The process of compression was followed with BAM which showed once more the coexistence of fluid and condensed domains (Figure 2 B). The BAM micrographs indicate that, in the presence of NSAIDs in the subphase, the domains characteristic for the coexistence of liquid expanded (LE) and liquid condensed (LC) states coalesce at slightly higher surface pressures compared to those of the lipid mixture in the absence of the drug, which is in accordance with the shift of the compression isotherms to higher surface pressures indicating the penetration of the drugs. It seems that the drugs are preferentially localized in the disordered phase since the condensed domains did not change.

Furthermore, comparing with the lipid mixture alone, BAM images (Figure 2 B) reflect the existence of less condensed domains in the case of ibuprofen (Figure $2 \mathrm{~B}$ - number $2 \mathrm{~b}$ shows less brighter spots than number $1 \mathrm{~b}$ ), whereas in the case of piroxicam the condensed domains 
are more evident than in the non-influenced monolayer (Figure $2 \mathrm{~B}$ - number 3a shows bigger brighter spots than number $1 b)$.

Moreover, it is also evident that the drugs are expelled from the monolayer at smaller molecular areas, and at $53 \AA^{2} /$ molecule the BAM images (Figure 2 B) look almost the same whether we are observing images of the lipid mixture alone (Figure $2 \mathrm{~B}$ - number 1d), or lipid mixture with the drug (Figure $2 \mathrm{~B}$ - numbers $2 \mathrm{~d}$ and $3 \mathrm{c}$ ). This is also visible on the isotherms (Figure $2 \mathrm{~A}$ - numbers $1 \mathrm{~d}, 2 \mathrm{~d}$ and $3 \mathrm{c}$ ) where the $\pi / \mathrm{A}$ plots from the lipid mixture and NSAIDs almost superimpose.

\subsection{NSAIDs binding studies to PLA}

Binding studies of NSAIDs to PLA 2 were performed to evaluate the contribution of the direct interaction between drugs and enzyme on the inhibitory effect of the enzymatic activity. The fluorescence of the aromatic region of $\mathrm{PLA}_{2}$, due to the existence of tryptophans, gives the possibility to evaluate the binding of the enzyme to a drug molecule [27]. The emission spectra of $\mathrm{PLA}_{2}$ were examined in the absence and presence of piroxicam and ibuprofen after 2, 5, 8 and 10 minutes reaction in a concentration range of $0-150 \mu \mathrm{M}$. For both drugs studied, a concentration dependent decrease of the fluorescence intensity was obtained and this quenching effect was time independent. The PLA 2 - drug binding constants were calculated by a graphical method where Langmuir equation was used to fit the fluorescence quenching intensities (expressed as $\%$ of quenching) according to the concentration of NSAID [27]:

$$
\text { Quenching }(\%)=\frac{\mathrm{y}_{\text {máx }}}{1+\frac{\mathrm{K}_{\mathrm{d}}}{\log [\mathrm{NSAID}]}}
$$


The values of $y_{\max }$ (maximum quenching value obtained) and $K_{d}$ (dissociation constant which is inversely proportional to the binding constant, $\mathrm{K}_{\mathrm{b}}$ ) are determined from fitting a plot of quenching ( $\%$ of fluorescence deactivation correspondent to the $\%$ of bound enzyme molecules) as a function of $\log [$ NSAID] (logarithm of NSAID concentration). A Langmuir isotherm plot illustrating the binding behaviour for piroxicam is presented on Figure 3. A similar plot was obtained for ibuprofen. For both drugs, the isotherm is an "S-shaped" curve with the drug concentration corresponding to the $\mathrm{K}_{\mathrm{d}}$ at the midpoint of the quasi-linear portion of the curve.

The binding constants to PLA 2 obtained for piroxicam and ibuprofen were $6.0 \times 10^{-2} \mu \mathrm{M}$ and $1.5 \times 10^{-2} \mu \mathrm{M}$, respectively, which indicates that piroxicam has a more extensive binding to the enzyme.

\subsection{Evaluation of PLA $A_{2}$ activity and its inhibition by the NSAIDs}

The inhibition of $\mathrm{PLA}_{2}$ enzyme can be evaluated by several methods. One of them is the determination of the amount of free fatty acid (FFA) that is released over time as a consequence of the enzyme hydrolytic activity against a lipidic substrate [28]. In this case the lipidic substrates were LUVs of DMPC/DPPE (7:3) and the chosen method for the quantification of the phospholipid hydrolysis was based on the use of a fluorescent probe (ADIFAB) with high binding affinity for FFA. According to this method, the concentration of FFA ([FFA]) was quantified at $432 \mathrm{~nm}$ using the following expression:

$$
[\mathrm{FFA}]=\mathrm{K}_{\mathrm{D}} \frac{\mathrm{F}-\mathrm{F}_{0}}{\mathrm{~F}_{\text {máx }}-\mathrm{F}}
$$

Where $\mathrm{F}$ are the values of relative fluorescence, $\mathrm{F}_{0}$ is the fluorescence obtained in the absence of the enzyme; $F_{\text {máx }}=F_{o} / 19.5$ and $K_{D}=0.34 \quad[18]$. 
After determining the FFA concentrations it was possible to calculate total fatty acid concentrations ([FA $]_{\text {TOTAL }}$ ) according to the expression:

$$
[\mathrm{FA}]_{\mathrm{TOTAL}}=\mathrm{K}_{\mathrm{P}} \frac{\mathrm{V}_{\mathrm{m}}}{\mathrm{V}_{\mathrm{a}}} \times[\mathrm{FFA}]+[\mathrm{FFA}]
$$

Where $\mathrm{K}_{\mathrm{P}}$ is the partition coefficient of FFA, $\mathrm{V}_{\mathrm{m}}$ is the volume of the membrane phase and $\mathrm{V}_{\mathrm{a}}$ is the volume of the aqueous phase [18].

The inhibition capacity of the NSAIDs tested was quantified according to the reduction of $[\mathrm{FA}]_{\text {TOTAL }}$ considering that $[\mathrm{FA}]_{\text {TOTAL }}$ is $100 \%$ in the absence of the inhibitors. Ibuprofen and piroxicam were tested for different concentrations $(0-20 \mu \mathrm{M})$ using two types of incubation. When the NSAIDs were incubated in LUVs before the addition of PLA $\mathrm{P}_{2}$, there was no visible alteration in the hydrolysis. On the other hand, when PLA $_{2}$ was incubated with NSAIDs before the addition to the liposomes, it was found that the enzyme inhibition occurred and was concentration dependent. Figure 4 shows $\mathrm{PLA}_{2}$ inhibition with increasing concentrations of piroxicam and ibuprofen plotted as a function of the total fatty acid concentration ([FA $]_{\text {TOTAL }}$ $\%)$ produced after 5 min reaction time.

From Figure 4 it is possible to conclude that the total fatty acid concentration reduced significantly with increasing concentrations of NSAIDs and a small concentration of $6 \mu \mathrm{M}$ was enough to provoke an extensive inhibition of $\mathrm{PLA}_{2}$ (correspondent to $60 \%$ and $50 \%$ of total fatty acid concentration obtained with piroxicam and ibuprofen, respectively). According to the extent of reduction of fatty acid produced, ibuprofen revealed to be a stronger inhibitor of $\mathrm{PLA}_{2}$.

Since $[\mathrm{FA}]_{\text {TOTAL }}$ reduction occurred only when the drugs were previously incubated with the enzyme this indicates that possibly these NSAIDs require to be in direct contact with the enzyme to be able to act as inhibitors. 
The PLA 2 activity and its inhibition by the NSAIDs studied was evaluated by another method that makes use of the molecular area/time dependency (A / t ) for testing whether a direct interference with the enzyme is playing a role in the inhibition process. Figure 5 shows a typical example of an isotherm (A / t ) obtained in the absence and in the presence of NSAID (piroxicam).

The lipid substrate was a monolayer of DMPC/DPPE (7:3) spread on the aqueous phase (Tris $10 \mathrm{mM} ; \mathrm{NaCl} 150 \mathrm{mM}, \mathrm{CaCl}_{2} 1 \mathrm{mM}, \mathrm{pH}$ 7.4). The initial molecular area (Figure 5 - point A) corresponds to the area occupied by the monolayer with fully expanded barriers. During compression, the area decreases steeply until a fixed pressure of $25 \mathrm{mN}$ is reached (Figure 5 point B). After waiting some time for stabilization of the monolayer, the enzyme was injected (alone or after incubation with NSAID) into the subphase (Figure 5 - point C). When the enzyme is active, and in the absence of drug (Figure 5 - Line 1), the hydrolysis of the monolayer occurs at a constant pressure, and as a result, the produced lysophospholipids are dissolved in the aqueous subphase and thus the molecular area of the monolayer decreases over time. In contrast, when the enzyme and drug are injected into the subphase (Figure 5 Line 2) the hydrolytic activity is inhibited and the phospholipid molecular area remains constant. More than two hours after the NSAIDs' inhibitory studies have been started another injection of $1 \times 10^{-3} \mathrm{M} \mathrm{PLA}_{2}$ (not incubated with the drug) was carried out (Figure 5 - point D). This injection reinforces the enzyme concentration and as a consequence, the phospholipid hydrolysis starts, as observed by the decrease of the molecular area (Figure 5 from point $\mathrm{D}$ to the end).

\subsection{Evaluation of PLA $\mathrm{A}_{2}$ conformational changes}


CD spectroscopy allowed the study of possible PLA 2 conformational changes. PLA P $_{2}$ spectrum shows two negative bands near $209 \mathrm{~nm}$ and $223 \mathrm{~nm}$ and a positive band at $195 \mathrm{~nm}$, typical of $\alpha$-helical conformation (Figure 6 A - black curve). The $[\theta]_{223} /[\theta]_{209}$ ratio is greater than 1 (1.18, Table 1) indicating superhelical twist associated with coiled-coils or other assemblies of helices [29]. The $n-\pi^{*}$ transition ( 220-nm CD band) is responsive to the $\alpha$-helical content, whereas the $\pi-\pi^{*}$ excitation band at $\sim 208 \mathrm{~nm}$ polarizes parallel to the helix axis and is sensitive to whether the $\alpha$-helix is single-stranded or is an interacting helix as in the case of the two-stranded coiled-coils [30].This is supported by the crystal structure of the enzyme [31]. Each NSAID was studied individually regarding its ability to modify the enzyme's secondary structure. The binding of ligands can affect the structure of enzymes and it has been previously reported the ability of inhibitors to change $\mathrm{PLA}_{2}$ conformation through the increase or decrease in the $\alpha$-helical content $[3,32,33]$. It is visible that $\mathrm{PLA}_{2} \mathrm{CD}$ spectra after addition of the NSAIDs show minor differences comparing with that from the enzyme alone, indicating lack of major changes in the secondary structure (Figure 6 A). The ellipticity ratio $[\theta]_{223} /[\theta]_{209}$ increases in the presence of NSAIDs particularly piroxicam, indicating a stronger interaction between the enzyme and this drug, which is supported by the higher binding constant found for this case (Table 1).

In buffer solution, the enzyme presents mainly an $\alpha$-helix conformation where as in the presence of liposomes, PLA 2 exhibits as well a spectrum characteristic of $\alpha$-helical, but with the band at $223 \mathrm{~nm}$ more pronounced, indicating higher helical content and $[\theta]_{223} /[\theta]_{209}$ ratio (Figure $6 \mathrm{~B}$ and Table 1) [34] [35]. The increase of the ellipticity ratio $[\theta]_{223} /[\theta]_{209}$ indicates helices with increased flexibility [34] [35] [36]. Therefore, CD data suggest an increased level of more flexible $\alpha$-helices in $\mathrm{PLA}_{2}$ when the enzyme is bound to its natural substrate, phospholipid membranes. 
In agreement to these results, destabilization of enzymatic helices upon membrane binding have been shown previously for several proteins or their fragments, such as cytochrome $\mathrm{c}, \alpha-$ lactalbumin, and the pore-forming domain of colicin resulting in structural changes in these proteins to more flexible conformations [12]. A possible mechanism for the formation of more flexible helices in $\mathrm{PLA}_{2}$ induced by binding to lipid bilayers may be disruption of a few intrahelical hydrogen bonds and formation of such bonds between the enzyme and the phosphate, carbonyl, and hydroxyl groups of membrane lipids. These results imply that during interfacial activation via membrane binding, the $\mathrm{PLA}_{2}$ molecules acquire more dynamic properties, which presumably confer plasticity to the enzyme that is required for the multistep catalytic process. This might render the enzyme more flexible and stabilize the transition-state complex which may contribute to enzyme activation and facilitate catalysis [12]. The incubation of liposomes with the drugs followed by the addition of PLA 2 provided spectra resembling the one of $\mathrm{PLA}_{2}$ only in presence of LUVs (data not shown). The incubation of $\mathrm{PLA}_{2}$ with piroxicam followed by the addition of liposomes showed similar tendency with slight increase of the absolute value of $[\theta]_{223}$ (Figure $6 \mathrm{~B}$ - dark gray curve). In the case of PLA 2 incubated first with ibuprofen and then with LUVs (Figure 6 B- light gray curve), the overall CD signal decreases and the noise increases, suggesting the occurrence of aggregation. The presence of aggregates is known to reduce the signal intensity due to light scattering and a shadowing effect [37]. The formation of aggregates upon interaction of PLA 2 with ibuprofen in the presence of liposomes can be correlated with the better stabilization of this NSAID in the lipid layer which might have implications on the interactions of the enzyme with the substrate.

The increase of the ratio $[\theta]_{223} /[\theta]_{209}$ is large in samples containing both liposomes and NSAIDs when compared to the samples with enzyme and drug or only liposomes indicating a pronounced alteration in the distorted structure of the enzyme (Table 1). 
A $[\theta]_{223} /[\theta]_{209}$ amplitude ratio near 1.5 rather than near 1 has been attributed to tryptophan contributions [38]. In general, pairwise or multiple coupling between any of the aromatic or other side-chain chromophores could lead to substantial effects in the 200-250-nm region and has been proposed to explain the unusual CD of fd Bacteriophage [39]. Considering that the intact tryptophan residues and certain level of helix distortion are essential for the enzymatic activity of $\mathrm{PLA}_{2}[40][39]$ the changes observed in the $[\theta]_{223} /[\theta]_{209}$ ratio induced by the drugs might be associated with the rearrangement of functional residues toward the interaction with phospholipids/substrate, and might be associated with these NSAIDs' capacity to inhibit the interaction of the enzyme with the lipid substrate. In fact, enzymes like PLA 2 from snake venom, that are known to bind well to zwitterionic surfaces, tend to have one or more tryptophans located on their interfacial surfaces taking active part in the interaction of the enzyme with the lipid membrane where these residues are inserted into the glycerol region of the bilayer [41].

\section{Conclusions}

Piroxicam and ibuprofen, the two NSAIDs chosen for this study, were able to inhibit sPLA 2 from Naja mossambica mossambica as proved by fluorescent measurements using ADIFAB. The interactions between drug molecules and biomembranes were studied since they could induce alterations in the lipid structure and modulate the enzymatic performance to different levels of activity. Results have shown that the drugs studied were able to adsorb or to penetrate into lipid monolayers due to their intrinsic surface activity. However, ibuprofen has been shown to be better stabilized in the lipid layer, which might be related with its capacity to affect the performance of lipid-modifying enzymes like sPLA 2 . 
The possibility of a direct effect of the anti-inflammatory agents on PLA $_{2}$ was also assessed. In the molecular area/time dependency it was visible that the enzyme ability to hydrolyze the monolayer was clearly decreased, resulting from the direct interaction $\mathrm{PLA}_{2}$ - antiinflammatory agents, latter regained by the excess of enzyme added to the subphase medium.

The enzyme secondary structure was also evaluated by CD spectroscopy. The increase observed in $[\theta]_{223} /[\theta]_{209}$ ratio caused by both anti-inflammatory agents and in particularly in the presence of liposomes indicates a change in the helical structure related to $\mathrm{PLA}_{2}$ flexibility when interacting with the model membrane. This might be in turn related to an alteration of the distorted structural elements that determine the interfacial activation competence of the enzyme. The ratio closer to 1.5 also indicates an alteration associated to tryptophans. Spectrofluorometric analysis showed the tryptophan quenching by both drugs and further confirmed that piroxicam and ibuprofen prevent the enzyme from binding to its lipid substrate, either by shielding the tryptophan residue(s) and limiting the access to the active site, or causing a conformational change of $\mathrm{PLA}_{2}$ structure reducing its ability for catalysis. In this regard, it is also possible to observe that piroxicam has demonstrated a higher binding constant to $\mathrm{PLA}_{2}$ trough the tryptophan residues, which has been further confirmed by CD measurements where the ellipticity $[\theta]_{223} /[\theta]_{209}$ ratio increases particularly in the presence of piroxicam, indicating a stronger interaction between this NSAID and the enzyme. Despite the smaller binding effect to the enzyme tryptophan residues, ibuprofen has proven to be a stronger inhibitor of $\mathrm{PLA}_{2}$ activity. In this case, the biophysical changes induced by this drug in the lipid interface seem to have their own contribution to the whole inhibition process. Indeed, upon interaction of $\mathrm{PLA}_{2}$ with ibuprofen in the presence of liposomes, the CD signal decreases and the noise increases, suggesting the occurrence of aggregation. This can be correlated with the better stabilization of ibuprofen in the lipid layer which, added to its binding capacity to the enzymatic tryptophan residues, might promote desorption of PLA 2 
from the substrate. In agreement to this, it has been reported that the surface properties of the membrane are a prerequisite for binding of $\mathrm{PLA}_{2}$ in a proper way, required for the induction of the conformational changes that ultimately activate the enzyme [42].

In conclusion, the results in the present study clearly show that the NSAIDs studied inhibit $\mathrm{PLA}_{2}$ and that the inhibition of interfacial enzymes should be studied by a combination of methods that are able to cover the enzyme conformational changes upon binding and their correlation with membrane biophysical effects, since these aspects can constitute synergistic determinants of the overall inhibitory effect. 


\section{Acknowledgements}

Partial financial support for this work was provided by FCT (Lisbon), through the contract PTDC/SAU-FCF/67718/2006. Diana Gaspar thanks FCT (Lisbon) for the fellowship (SFRH/BD/29308/2006). 


\section{References:}

[1] N. Singh, T. Jabeen, R.K. Somvanshi, S. Sharma, S. Dey, T.P. Singh, Phospholipase $\mathrm{A}(2)$ as a target protein for nonsteroidal anti-inflammatory drugs (NSAIDs): Crystal structure of the complex formed between phospholipase A(2) and oxyphenbutazone at 1.6 angstrom resolution, Biochemistry. 43 (2004) 14577-14583.

[2] D.A. Six, E.A. Dennis, The expanding superfamily of phospholipase A(2) enzymes: classification and characterization, Biochim. Biophys. Acta, Mol. Cell Biol. Lipids. 1488 (2000) 1-19.

[3] N.M. Jameel, M.A. Shekhar, B.S. Vishwanath, alpha-lipoic acid: An inhibitor of secretory phospholipase A(2) with anti-inflammatory activity, Life Sciences. 80 (2006) 146153.

[4] M.C. Monti, A. Casapullo, C. Santomauro, M.V. D'Auria, R. Riccio, L. GomezPaloma, The molecular mechanism of bee venom phospholipase $\mathrm{A}(2)$ inactivation by bolinaquinone, Chembiochem. 7 (2006) 971-980.

[5] R.H. Schaloske, E.A. Dennis, The phospholipase A(2) superfamily and its group numbering system, Biochim. Biophys. Acta, Mol. Cell Biol. Lipids. 1761 (2006) 1246-1259.

[6] C. Raguenes-Nicol, F. Russo-Marie, G. Domage, N. Diab, E. Solito, F. Dray, et al., Anti-inflammatory mechanism of alminoprofen: Action on the phospholipid metabolism pathway, Biochem. Pharmacol. 57 (1999) 433-443.

[7] S. Yedgar, Y. Cohen, D. Shoseyov, Control of phospholipase A(2) activities for the treatment of inflammatory conditions, Biochim. Biophys. Acta, Mol. Cell Biol. Lipids. 1761 (2006) 1373-1382.

[8] R.M. Kini, Excitement ahead: structure, function and mechanism of snake venom phospholipase A(2) enzymes, Toxicon. 42 (2003) 827-840. 
[9] K. Wagner, B. Desbat, G. Brezesinski, Liquid-liquid immiscibility in model membranes activates secretory phospholipase A(2), Biochim. Biophys. Acta, Biomembr. 1778 (2008) 166-174.

[10] U. Dahmen-Levison, G. Brezesinski, H. Mohwald, Specific adsorption of PLA(2) at monolayers, Thin Solid Films. 329 (1998) 616-620.

[11] K. Wagner, G. Brezesinski, Modifying dipalmitoylphosphatidylcholine monolayers by n-hexadecanol and dipalmitoylglycerol, Chem. Phys. Lipids. 145 (2007) 119-127.

[12] S.A. Tatulian, R.L. Biltonen, L.K. Tamm, Structural changes in a secretory phospholipase $\mathrm{A}(2)$ induced by membrane binding: A clue to interfacial activation?, J. Mol. Biol. 268 (1997) 809-815.

[13] W.R. Burack, M.E. Gadd, R.L. Biltonen, Modulation of Phospholipase A(2) Identification of an inactive membrane-bound state, Biochemistry. 34 (1995) 14819-14828.

[14] U. Dahmen-Levison, G. Brezesinski, H. Mohwald, J. Jakob, P. Nuhn, Investigations of lipid-protein interactions on monolayers of chain-substituted phosphatidylcholines Angew. Chem. Int. Ed. Engl. 39 (2000) 2775-2778.

[15] E.J. Shimshick, H.M. McConnell, Lateral phase separation in phospholipid membranes, Biochemistry. 12 (1973) 2351-2360.

[16] R.C. Franson, D. Eisen, R. Jesse, C. Lanni, Inhibition of Highly Purified Mammalian Phospholipases a2 by Non-Steroidal Anti-Inflammatory Agents - Modulation by CalciumIons, Biochem. J. 186 (1980) 633-636.

[17] I.B. Lobo, J.R.S. Hoult, Group-I, Group-II and Group-III Extracellular Phospholipases A(2) - Selective-inhibition of group-II enzymes by Indomethacin but not other NSAIDs, Agents and Actions. 41 (1994) 111-113. 
[18] G.V. Richieri, R.T. Ogata, A.M. Kleinfeld, The measurement of free fatty acid concentration with the fluorescent probe ADIFAB: A practical guide for the use of the ADIFAB probe, Mol. Cell. Biochem. 192 (1999) 87-94.

[19] G.V. Richieri, R.T. Ogata, A.M. Kleinfeld, Thermodynamics of Fatty-Acid-Binding to Fatty-Acid-Binding Proteins and Fatty-Acid Partition between Water and Membranes Measured Using the Fluorescent-Probe Adifab, J. Biol. Chem. 270 (1995) 15076-15084.

[20] K. Wagner, G. Brezesinski, Phospholipase D activity is regulated by product segregation and the structure formation of phosphatidic acid within model membranes, Biophys. J. 93 (2007) 2373-2383.

[21] V. Melzer, G. Weidemann, D. Vollhardt, G. Brezesinski, R. Wagner, B. Struth, et al., Brewster angle microscopy and X-ray GID studies of morphology and crystal structure in monolayers of N-tetradecyl-gamma,delta-dihydroxypentanoic acid amide, J. Phys. Chem. B. 101 (1997) 4752-4758.

[22] J.R. Lakowicz, Principles of Fluorescence Spectroscopy (Springer, New York, 2006).

[23] X.H. Zhai, J.B. Li, G. Brezesinski, Q. He, H. Mohwald, L.H. Lai, et al., Direct observations of the cleavage-reaction of an L-DPPC monolayer catalyzed by phospholipase A(2) and inhibited by an indole inhibitor at the air/water interface, Chembiochem. 4 (2003) 299-305.

[24] E. Jablonowska, R. Bilewicz, Interactions of ibuprofen with Langmuir monolayers of membrane lipids, Thin Solid Films. 515 (2007) 3962-3966.

[25] K.V. Damodaran, K.M. Merz, Jr., A comparison of DMPC- and DLPE-based lipid bilayers, Biophys. J. 66 (1994) 1076-1087.

[26] P.B. Welzel, H.K. Cammenga, Equilibrium penetration of DMPC monolayers by sodium cholate, J. Colloid Interf. Sci. 207 (1998) 70-77. 
[27] R.A. Copeland, Enzymes: a practical introduction to structure, mechanism, and data analysis (Wiley-VCH, 2000).

[28] G.V. Richieri, A.M. Kleinfeld, Continuous measurement of Phospholipase A(2) activity using the fluorescent probe ADIFAB, Anal. Biochem. 229 (1995) 256-263.

[29] S.Y.M. Lau, A.K. Taneja, R.S. Hodges, Synthesis of a model protein of defined secondary and quaternary structure - effect of chain length on the stabilization and formation of 2-stranded alpha-helical coiled-coils, J. Biol. Chem. 259 (1984) 3253-3261.

[30] T.M. Cooper, R.W. Woody, The effect of conformation on the CD of interacting helices: a theoretical study of tropomyosin, Biopolymers. 30 (1990) 657-676.

[31] R.K. Arni, R.J. Ward, Phospholipase A(2) - A structural review, Toxicon. 34 (1996) $827-841$.

[32] N.M. Jameel, B.M. Frey, F.J. Frey, T.V. Gowda, B.S. Vishwanath, Inhibition of secretory phospholipase $\mathrm{A}(2)$ enzyme by bilirubin: A new role as endogenous antiinflammatory molecule, Mol. Cell. Biochem. 276 (2005) 219-225.

[33] Y.H. Lin, W.N. Huang, S.C. Lee, W.G. Wu, Heparin reduces the alpha-helical content of cobra basic phospholipase $\mathrm{A}(2)$ and promotes its complex formation, Int. J. Biol. Macromol. 27 (2000) 171-176.

[34] S.A. Tatulian, Structural effects of covalent inhibition of phospholipase A(2) suggest allosteric coupling between membrane binding and catalytic sites, Biophys. J. 84 (2003) $1773-1783$.

[35] N.E. Zhou, C.M. Kay, R.S. Hodges, Synthetic model proteins - positional effects of interchain hydrophobic interactions on stability of 2-stranded alpha-helical coiled-coils, J. Biol. Chem. 267 (1992) 2664-2670. 
[36] S. Qin, A.H. Pande, K.N. Nemec, S.A. Tatulian, The N-terminal alpha-helix of pancreatic phospholipase $\mathrm{A}(2)$ determines productive-mode orientation of the enzyme at the membrane surface, J. Mol. Biol. 344 (2004) 71-89.

[37] L.N. Duysens, The flattening of the absorption spectrum of suspensions, as compared to that of solutions, Biochim. Biophys. Acta. 19 (1956) 1-12.

[38] G.E. Arnold, L.A. Day, A.K. Dunker, Tryptophan contributions to the unusual circular-dichroism of Bacteriophage-Fd, Biochemistry. 31 (1992) 7948-7956.

[39] L.S. Chang, K.W. Kuo, C.C. Chang, Identification of functional involvement of tryptophan residues in Phospholipase-A(2) from Naja-Naja-Atra (Taiwan Cobra) snakevenom, Biochim. Biophys. Acta. 1202 (1993) 216-220.

[40] M. Sumandea, S. Das, C. Sumandea, W. Cho, Roles of aromatic residues in high interfacial activity of Naja naja atra phospholipase A(2), Biochemistry. 38 (1999) 1629016297.

[41] J.M. Winget, Y.H. Pan, B.J. Bahnson, The interfacial binding surface of phospholipase A2s, Biochim. Biophys. Acta, Mol. Cell Biol. Lipids. 1761 (2006) 1260-1269.

[42] S.A. Tatulian, Toward understanding interfacial activation of secretory phospholipase A2 (PLA2): membrane surface properties and membrane-induced structural changes in the enzyme contribute synergistically to PLA2 activation, Biophys. J. 80 (2001) 789-800. 


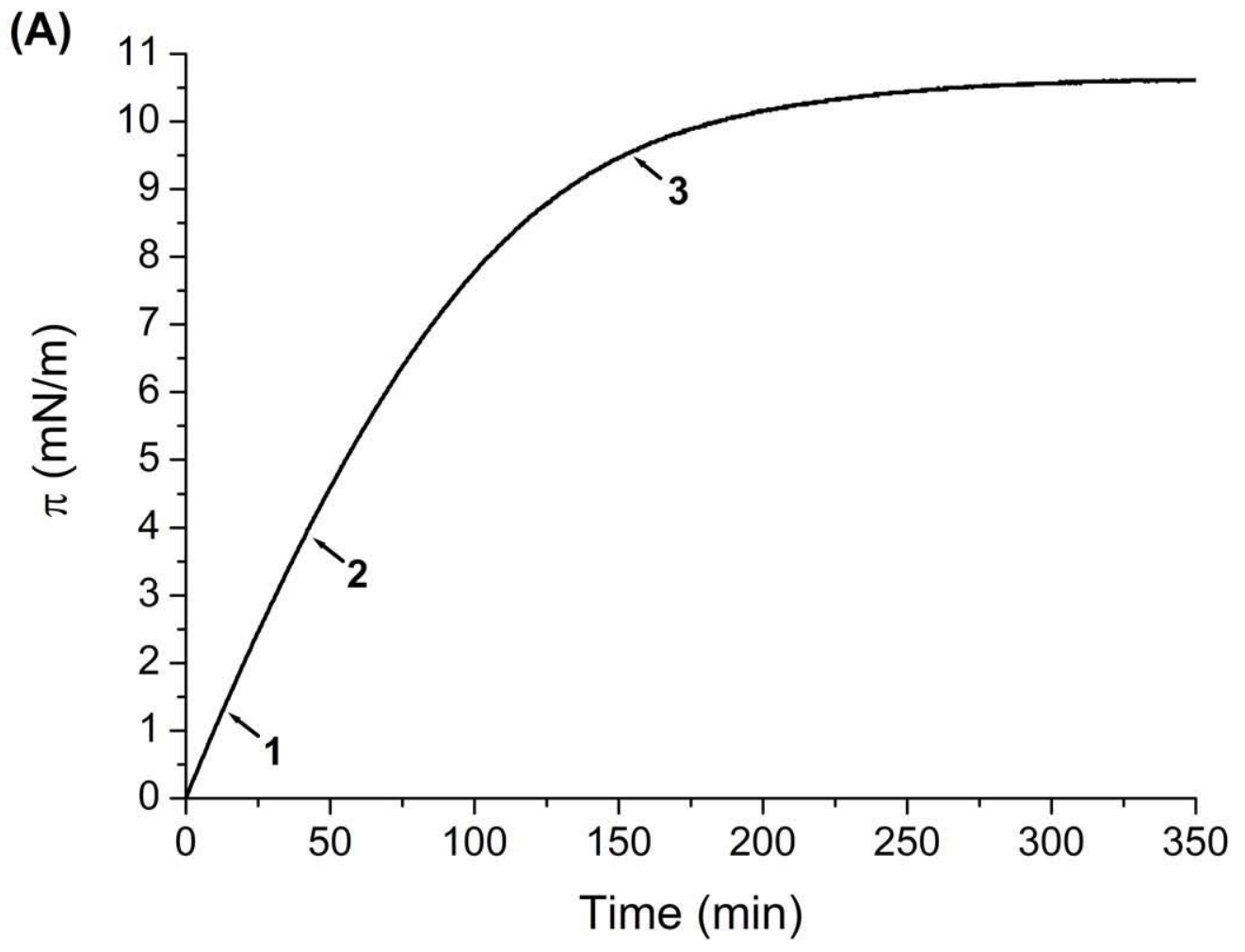

(B)

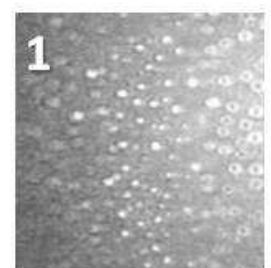

14 minutes

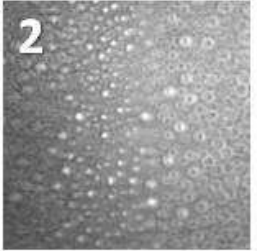

43 minutes

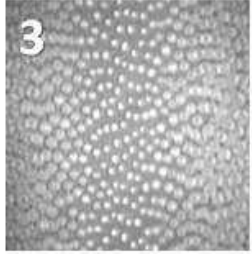

155 minutes

Figure 1. (A) Surface pressure as a function of time of DMPC/DPPE (7:3) monolayer on a buffered subphase containing piroxicam $(20 \mu \mathrm{M})$ and (B) BAM images showing the 
morphology of the mixed monolayer at different time periods. The images size is $280 \times 300$ $\mu \mathrm{m}^{2}$. 


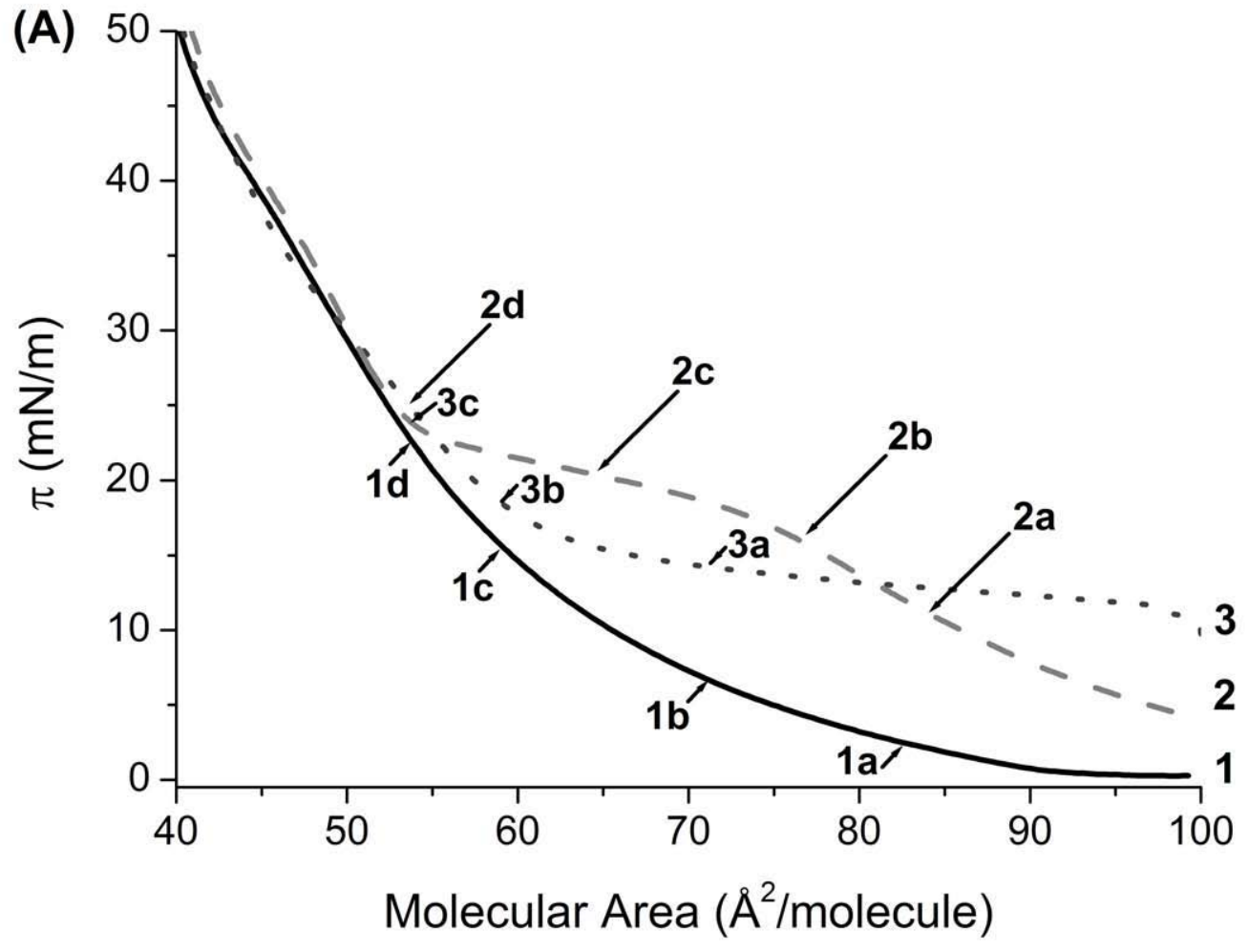

(B)

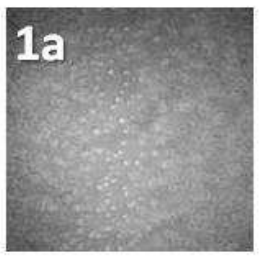

$82 \AA^{2} /$ molecule

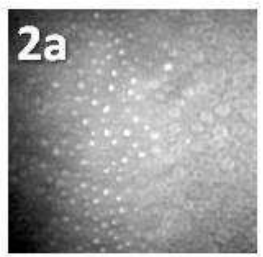

$84 \AA^{2} /$ molecule

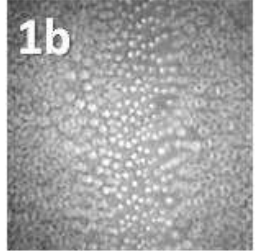

$73 \AA^{2} /$ molecule

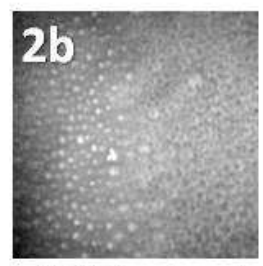

$76 \AA^{2} /$ molecule

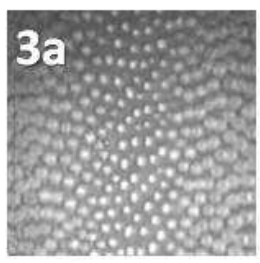

$69 \AA^{2} /$ molecule

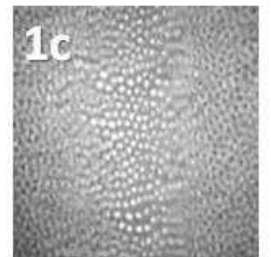

$59 \AA^{2} /$ molecule

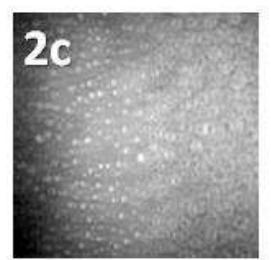

$64 \AA^{2} /$ molecule

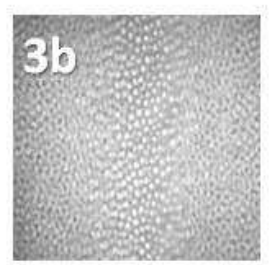

$59 \AA^{2} /$ molecule

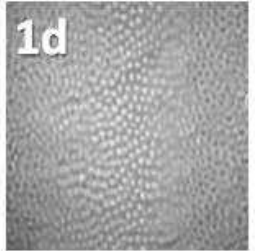

$53 \AA^{2} /$ molecule

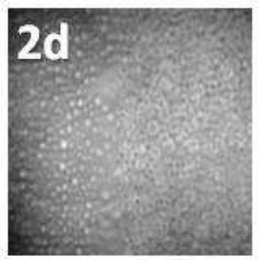

$53 \AA^{2} /$ molecule

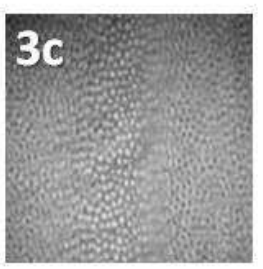

$53 \AA^{2} /$ molecule

Figure 2. (A) Surface pressure/area isotherms of DMPC/DPPE (7:3) monolayers on a buffered subphase (Tris $10 \mathrm{mM}, \mathrm{NaCl} 150 \mathrm{mM}, \mathrm{Ca}^{2+} 1 \mathrm{mM}, \mathrm{pH} 7.4$ ) (Line 1) and on a 
buffered subphase containing $20 \mu \mathrm{M}$ of ibuprofen (Line 2) or of piroxicam (Line 3) and respective (B) BAM images (first row on buffer, second row on ibuprofen and third row on piroxicam solutions). The images size is $280 \times 300 \mu \mathrm{m}^{2}$. 


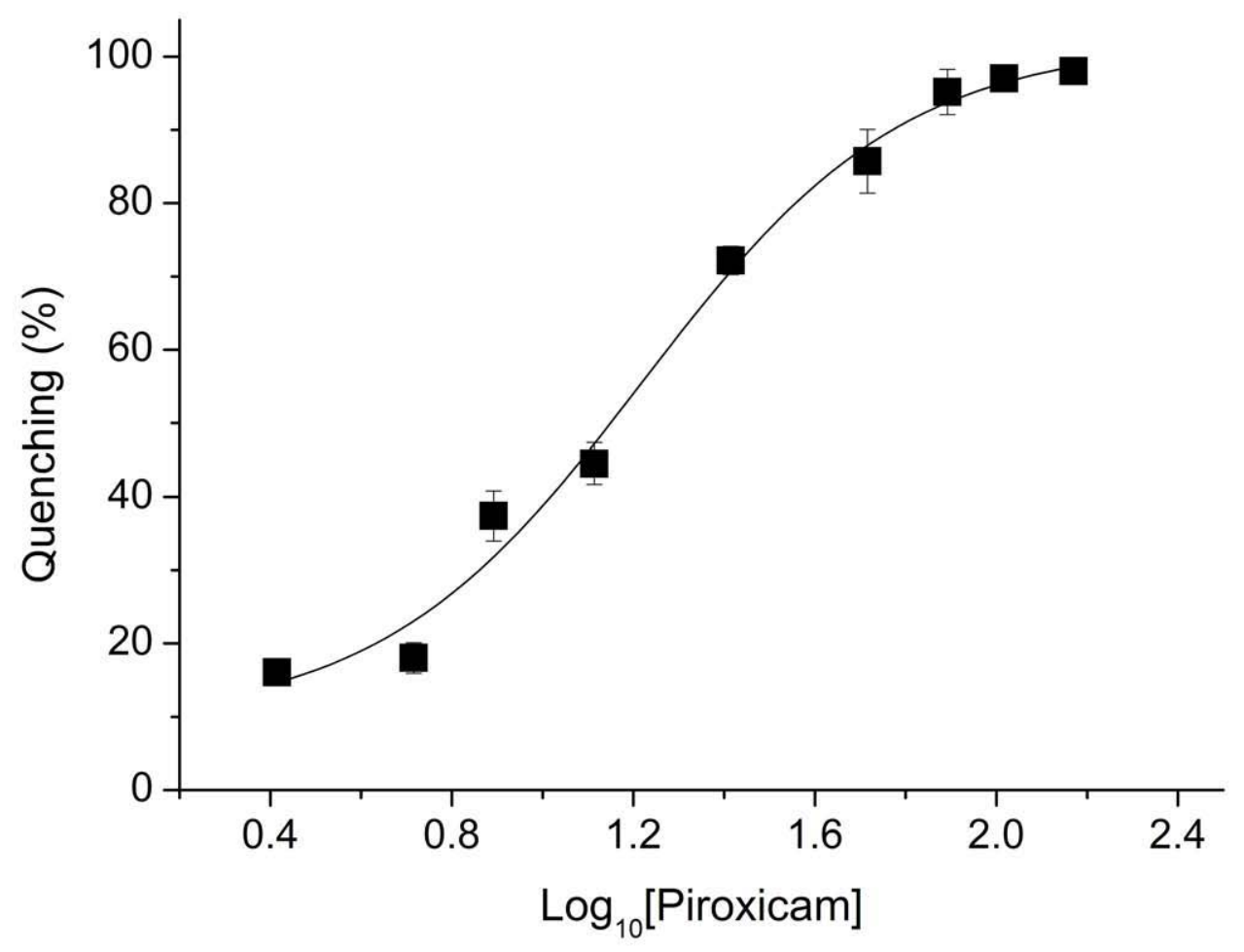

Figure 3. Langmuir plot for $\mathrm{PLA}_{2}$-piroxicam binding obtained from the fitting of $\%$ Quenching of $\mathrm{PLA}_{2}$ fluorescence as a function of piroxicam concentrations. Values represent the mean of three experiments. 


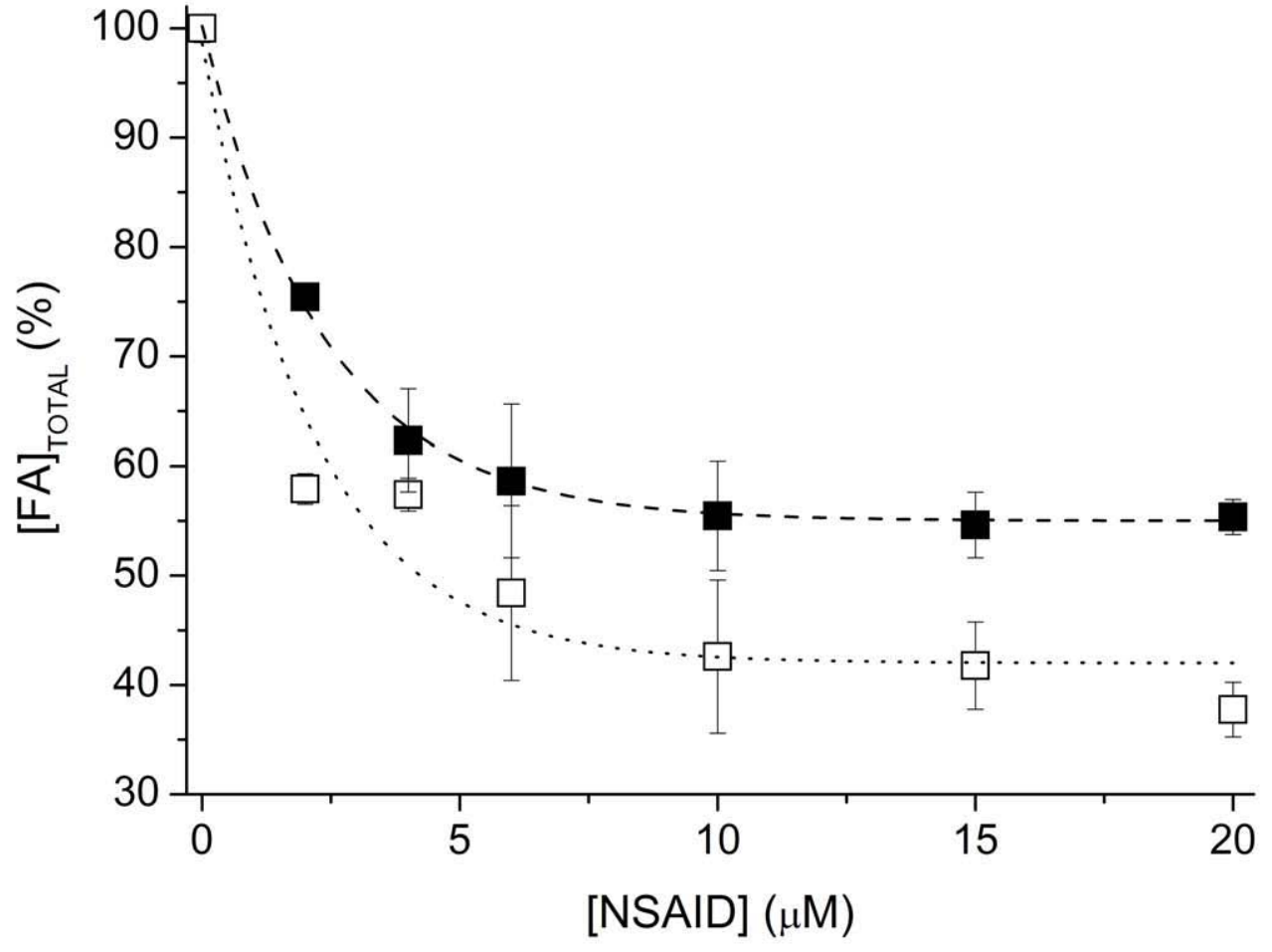

Figure 4. Fatty acid concentration (\%) obtained after 5 min hydrolysis reaction catalyzed by $\mathrm{PLA}_{2}$ in DMPC/DPPE (7:3) LUVs after incubation of the enzyme with piroxicam ( $\left.\mathbf{\square}\right)$ or ibuprofen ( $\square$ ). Values represent the mean of three experiments. 


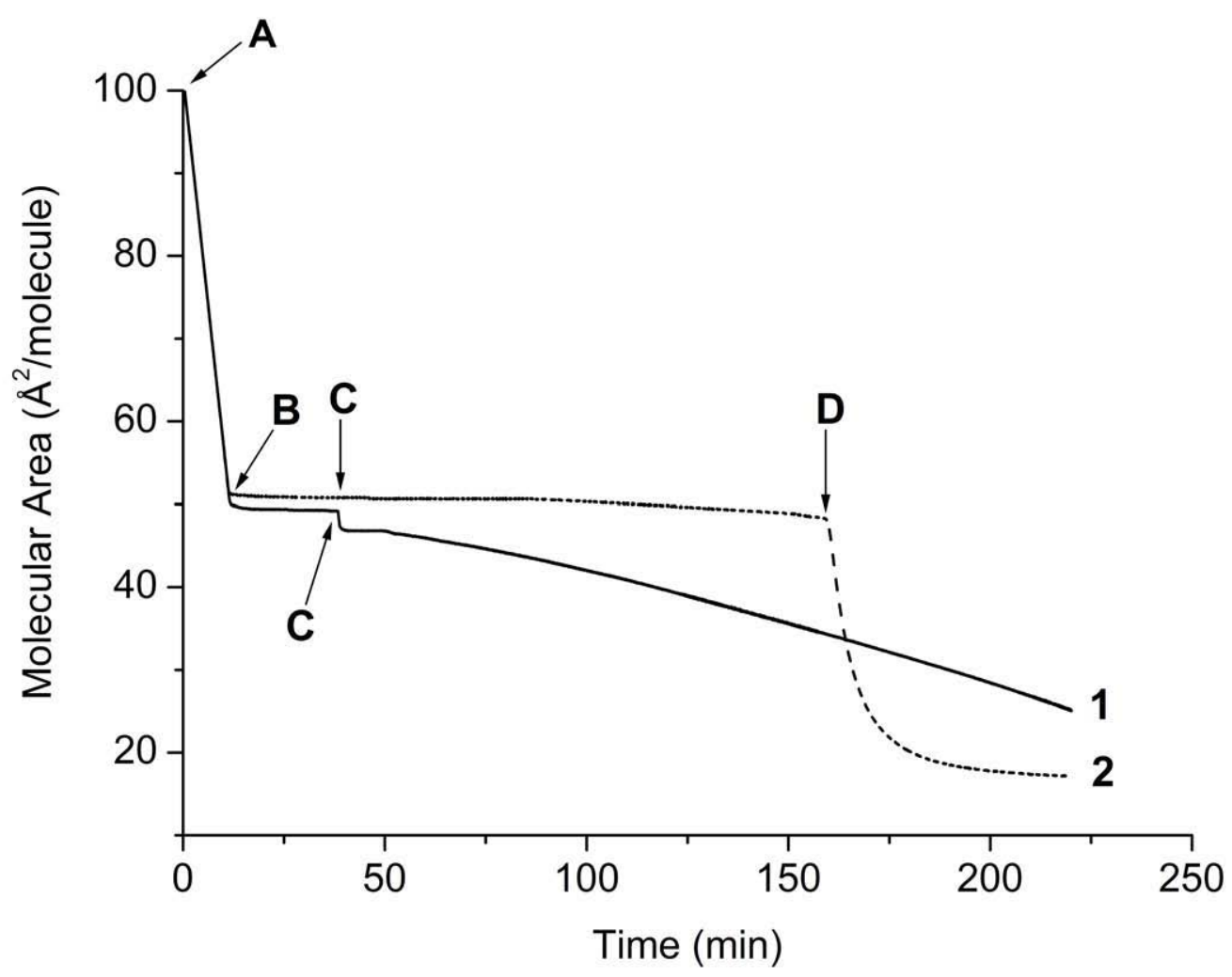

Figure 5. Area/time isotherm of a DMPC/DPPE (7:3) monolayer showing the hydrolysis reaction catalyzed by $\mathrm{PLA}_{2}$ in the absence (line 1) and presence (line 2) of $50 \mathrm{nM}$ piroxicam (Tris $10 \mathrm{mM}$; $\mathrm{NaCl} 150 \mathrm{mM} ; \mathrm{Ca}^{2+} 1 \mathrm{mM}$; pH 7.4). The initial molecular area (Figure 5 - point A) corresponds to the area occupied by the monolayer with fully expanded barriers. During compression, the area decreases until a fixed pressure of $25 \mathrm{mN} / \mathrm{m}$ is reached (Figure 5 - point B). After waiting some time for stabilization of the monolayer, the enzyme was injected (4 $\mu \mathrm{M})$ (alone or after incubation with NSAID) into the subphase (Figure 5 - point $\mathrm{C}$ ). Point D indicates the moment of another PLA 2 injection $\left(1 \times 10^{-3} \mathrm{M}\right)$. 


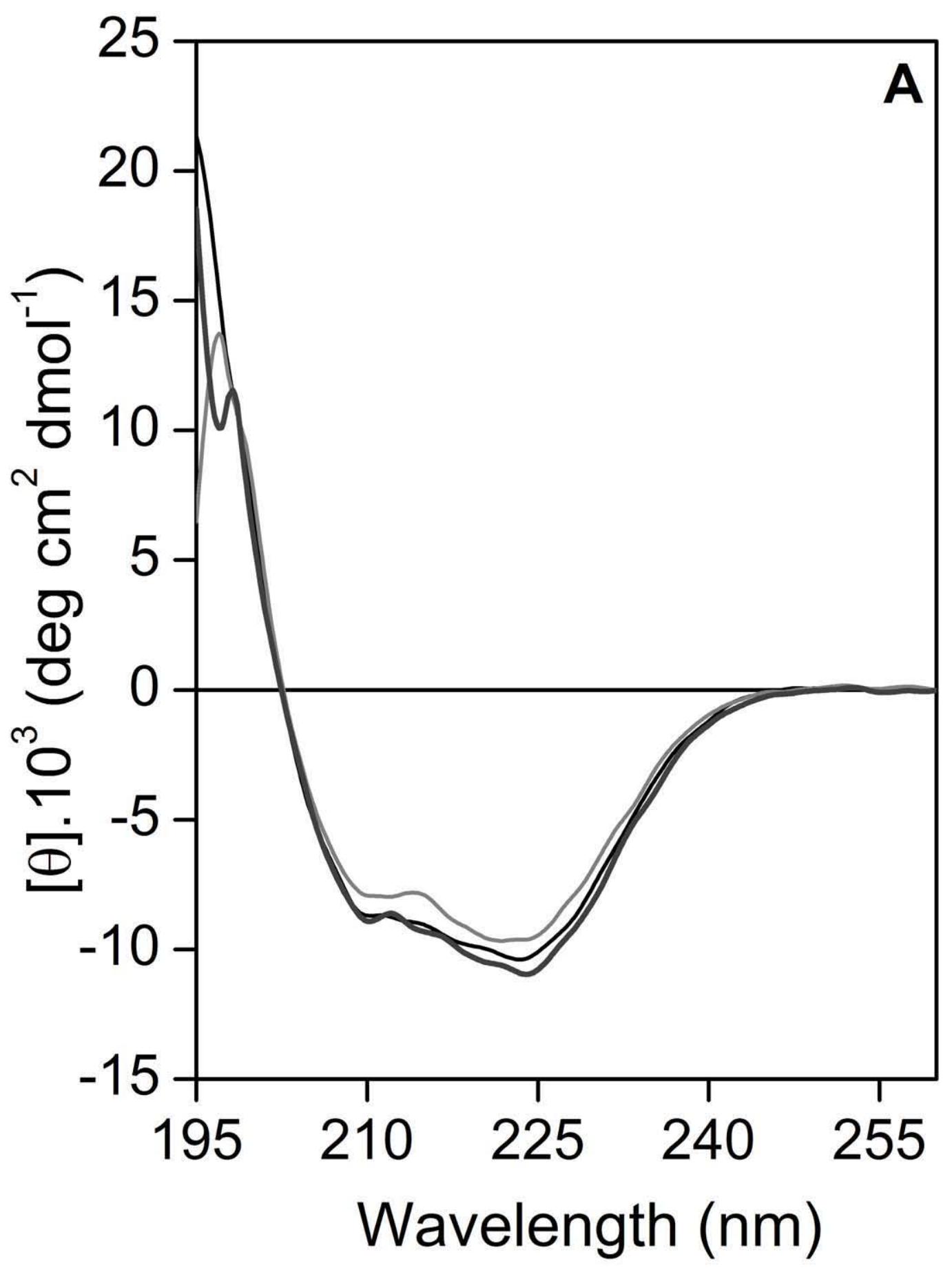




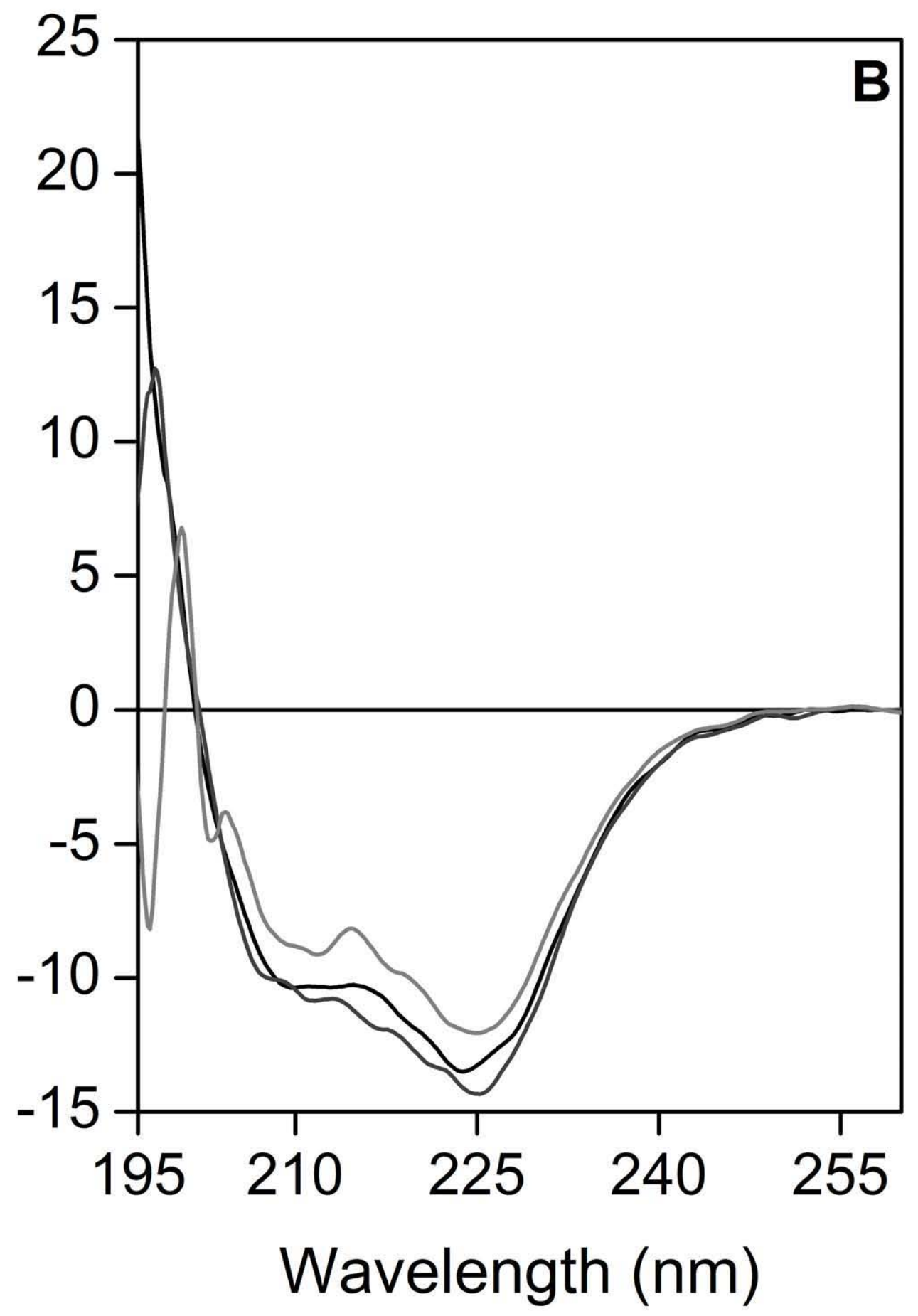

Figure 6. (A) CD spectra of $\mathrm{PLA}_{2}(8.9 \mu \mathrm{M})$ alone (black curve); with piroxicam (dark gray curve); or with ibuprofen (light gray curve). (B) CD spectra of $\operatorname{PLA}_{2}(8.9 \mu \mathrm{M})$ after addition of LUVs of DMPC/DPPE (7:3) (black curve) and CD spectra of $\operatorname{PLA}_{2}(8.9 \mu \mathrm{M})$ that was first mixed with NSAIDs (piroxicam (dark gray curve) or ibuprofen (light gray curve)) and then added to the liposomes. The noise below $200 \mathrm{~nm}$ is due to the $\mathrm{NaCl}$ in the solution. 
Table 1 Ellipticities and $[\theta]_{223} /[\theta]_{209}$ ratio of $\mathrm{PLA}_{2}$

$$
[\theta]_{223}\left(\times 10^{3} \mathrm{deg} \mathrm{\textrm {cm } ^ { 2 }} \mathrm{dmole}^{-1}\right) \quad[\theta]_{223} /[\theta]_{209}
$$

$\begin{array}{lcc}\mathrm{PLA}_{2} & -11.0 & 1.18 \\ \mathrm{PLA}_{2}+\text { Piroxicam } & -10.6 & 1.30 \\ \mathrm{PLA}_{2}+\text { ibuprofen } & -9.8 & 1.25 \\ \mathrm{PLA} & +\mathrm{LUV} & 1.29 \\ \mathrm{PLA}_{2}+\text { Piroxicam + LUV } & -12.6 & 1.35 \\ & -13.7 & \\ \mathrm{PLA}_{2}+\text { Ibuprofen + LUV } & -11.8 & 1.36\end{array}$




\section{Research highlights}

- Piroxicam and ibuprofen are able to inhibit SPLA $_{2}$ from Naja mossambica mossambica.

- Piroxicam and ibuprofen prevent the enzyme from binding to its lipid substrate, either by shielding the tryptophan residue(s) and limiting the access to the active site, or causing a conformational change of $\mathrm{PLA}_{2}$ structure reducing its ability for catalysis.

- Ibuprofen has been shown to be better stabilized in the lipid layer affecting the performance of $\mathrm{sPLA}_{2}$ and being as a result a stronger inhibitor of its activity. 